\title{
Pathways of bond topology transitions at the interface of silicon nanocrystals and amorphous silica matrix
}

\author{
D. E. Yilmaz* and C. Bulutay ${ }^{\dagger}$ \\ Department of Physics, Bilkent University, Ankara 06800, Turkey \\ T. Çă̆ı̆ \\ Artie McFerrin Department of Chemical Engineering, Texas A\&M University, Jack E. Brown Engineering Building, 3122 TAMU, \\ College Station, Texas 77843-3122, USA
}

(Received 7 January 2008; revised manuscript received 29 February 2008; published 4 April 2008)

\begin{abstract}
The interface chemistry of silicon nanocrystals (NCs) embedded in an amorphous oxide matrix is studied through molecular dynamics simulations with the chemical environment described by the reactive force field model. Our results indicate that the $\mathrm{Si} \mathrm{NC}$-oxide interface is more involved than the previously proposed schemes, which were based on solely simple bridge or double bonds. We identify different types of threecoordinated oxygen complexes that are previously not noted. The abundance and the charge distribution of each oxygen complex is determined as a function of the NC size as well as the transitions among them. The oxidation at the surface of $\mathrm{NC}$ induces tensile strain to $\mathrm{Si}-\mathrm{Si}$ bonds, which become significant only around the interface, while the inner core remains unstrained. Unlike many earlier reports on the interface structure, we do not observe any double bonds. Furthermore, our simulations and analysis reveal that the interface bond topology evolves among different oxygen bridges through these three-coordinated oxygen complexes.
\end{abstract}

DOI: 10.1103/PhysRevB.77.155306

PACS number(s): 61.46.Hk, 68.35.Ct

\section{INTRODUCTION}

After a long arduous effort, photoluminescence from silicon has been achieved from its nanocrystalline form. ${ }^{1} \mathrm{~A}$ critical debate, however, continues over the nature of the interface chemistry of silicon nanocrystals ( $\mathrm{Si} \mathrm{NCs}$ ) embedded in amorphous silica, which has direct implications on the optical activity of the interface. ${ }^{2-6}$ Wolkin et al. reported that the oxidation of porous silicon quantum dots results in a redshift in the photoluminescence (PL) spectra, which indicates the importance of oxygen-related interface bond toplogy. ${ }^{2}$ Along this line, Puzder et al. compared PL calculations of nanoclusters with different passivants and surface configurations and proposed the main reason for the redshift to be double $\mathrm{Si}=\mathrm{O}$ bonds. ${ }^{3}$ Countering this, Luppi et al. reported excitonic luminescence features caused by $\mathrm{Si}-\mathrm{O}-\mathrm{Si}$ bridge bonds at the surface of silicon nanoclusters. ${ }^{4}$ As a supporting evidence for the latter, Gatti and Onida recently demonstrated that the $\mathrm{Si}-\mathrm{O}-\mathrm{Si}$ bond is the most stable isomer configuration. ${ }^{5}$ To reconcile, Vasiliev et al. claimed that the bridge bonds and double bonds have similar effects on PL. ${ }^{6}$

All of the work cited above represent density functional theory (DFT)-based calculations with small Si clusters of less than 100 atoms surrounded by either passivants such as hydrogen ${ }^{3-5}$ or oxygen., ${ }^{2,3}$ However, actual samples are profoundly different: the fabricated systems consist of Si NC with a diameter larger than $1 \mathrm{~nm}$, embedded in an amorphous silica $\left(a-\mathrm{SiO}_{2}\right)$ matrix. Identifying this fact, $\mathrm{Tu}$ and Tersoff studied the $\mathrm{Si} / a$-SiO2 interface using a bond order dependent empirical potential and proposed that $\mathrm{Si}-\mathrm{O}-\mathrm{Si}$ bridge bonds are the main blocks at the interface lowering the surface energy. ${ }^{7}$ By using the same model potential, Hadjisavvas and Kelires studied Si NCs embedded in $a-\mathrm{SiO}_{2}$ and also reported bridge bonds as the mechanism for lowering surface strain energy. ${ }^{8}$ In our previous work on the formation and structure of Si NCs, we also observed that this model potential falls short in characterizing the system accurately, especially the structure and chemistry at the interface. ${ }^{9}$ As a rigorous approach to interface structure and dynamics, Pasquarello et al. used first-principles molecular dynamics (MD) technique to investigate the planar $\mathrm{Si} / \mathrm{SiO}_{2}$ interface. ${ }^{10}$ They observed that the oxygen atoms are momentarily bonded to three silicon atoms during the oxidation process. The net effect of these threefold-coordinated oxygen atoms during the oxidation process was to expel silicon atoms out of the interface. This is interpreted as a balancing process to decrease the increased density of the $\mathrm{Si} / \mathrm{SiO}_{2}$ interface due to oxidation. ${ }^{10}$ However, no substantial breakthrough was made over the past decade on this issue.

\section{METHOD}

In this work, we employ the reactive force field (ReaxFF) model developed by van Duin et al., ${ }^{12}$ which improves Brenner's reactive bond order model ${ }^{11}$ to a level of accuracy and validity, allowing molecular dynamics simulations of the full reaction pathways in bulk. The parameters for this force field were obtained from fitting to the results of $a b$ initio calculations on relevant species as well as periodic boundary condition DFT-based calculations of various crystalline polymorphs of relevant materials. The ReaxFF calculates bond orders, which are the measure of bond strength from local geometry. This allows realistic chemical environment such as over- or undercoordination and bond breaking or formation for large-scale (about 5000 atoms) MD simulations.

To facilitate our discussion regarding the surface-bonded oxygen complexes, we distinguish among three different types of silicon atoms. We label those silicon atoms with all silicon neighbors each with zero oxidation state as $c$ to denote core silicon atoms. Among the remaining (non-c) sili- 


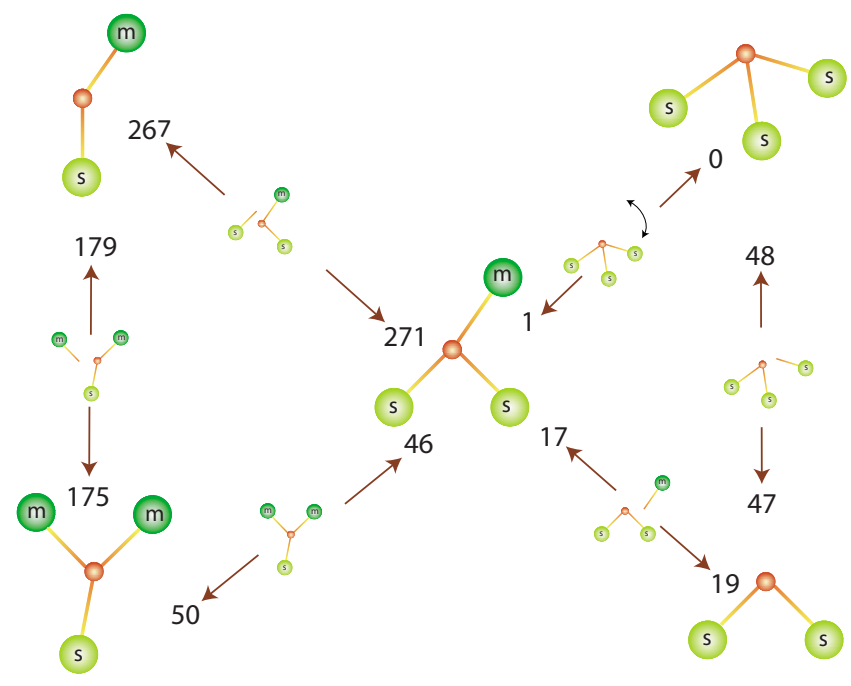

FIG. 1. (Color online) The transitions between different oxygen complexes bonded to the interface. The dark green (dark gray) large spheres represent matrix silicon atoms, the light green (light gray) large spheres represent surface silicon atoms of the $\mathrm{NC}$, and the small red (dark gray) spheres represent oxygen atoms. The numbers indicate the number of transitions recorded in the simulation in each direction among the complexes for the NC with a radius of $13.4 \AA$.

con atoms, those with at least one bond to $c$ are labeled as $s$, denoting surface silicon atoms. For further investigation of $\mathrm{NC}$, we seperate core $\mathrm{Si}$ atoms into two subcategories as inner-core and outer-core atoms: Among core $\mathrm{Si}$ atoms which have at least one surface Si neighbor categorized as outer-core $\mathrm{Si}$ atoms and rest of core $\mathrm{Si}$ atoms categorized as inner-core Si atoms. Finally, any other silicon atom is labeled as $m$, denoting a matrix silicon atom. Hence, a complex consisting of an oxygen atom bonded with two surface silicon atoms is labeled as ss. The other oxygen complexes are sm, ssm, sss, and mms, as sketched in Fig. 1, where the last three correspond to three-coordinated oxygen $(3 \mathrm{cO})$ atoms.

We use ReaxFF to represent the interactions in the model system. We start with a large simulation cell (box length of $43 \AA$ ) of silica glass formed through a melting and quenching process used by one of us ${ }^{13,14}$ earlier to study silica glasses. Next, similar to Hadjisavvas and Kelires, ${ }^{8}$ we delete all atoms within a predetermined radius to insert crystalline silicon to form NC. In this way, we create NCs with radii ranging from 5.5 to $16.7 \AA$. For the largest NC, we insert $967 \mathrm{Si}$ atoms into a spherical hole with a radius of $16.7 \AA$ created in an amorphous matrix. Even for this case, the minimum distance between the NC surface to the simulation box face is about $5 \AA$, which can still accommodate the interface layer. We also pay special attention in the removal of spherical region so that the correct stoichiometry for the amorphous matrix is met. Thus, our amorphous matrix has two $\mathrm{O}$ atoms for every $\mathrm{Si}$ atom with a density of $2.17 \mathrm{~g} / \mathrm{cm}^{3}$, which is the density of glass at room temperature and atmospheric pressure.

We set periodic boundary conditions in all directions, and while keeping NC at $100 \mathrm{~K}$, we employ a simulated annealing process to the $\mathrm{SiO}_{2}$ region to end up with an amorphous matrix free of artificial strain around the NC. Then, we set

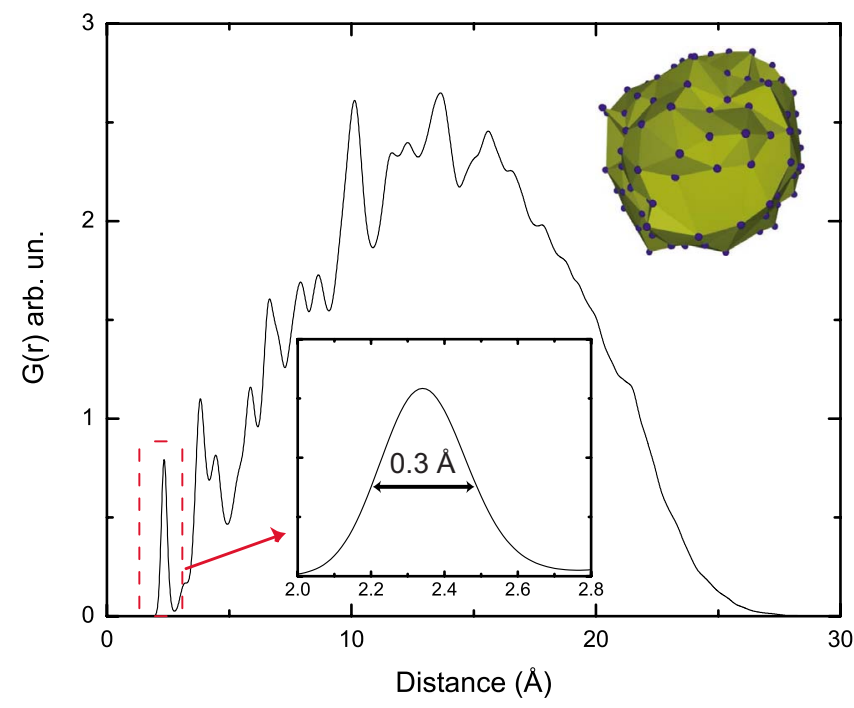

FIG. 2. (Color online) Radial distribution function of Si atoms in NC. Inset: The first peak resembles $\mathrm{Si}-\mathrm{Si}$ bond length distribution centered at around $2.34 \AA$ with a $0.3 \AA$ FWHM value. The second peak's width resembles bond angle deviation. Inset: Representation of NC surface created with Delaunay triangulation. Blue (dark gray) dots represents surface silicon atoms.

the whole systems' temperature to room temperature (300 K) and continue performing MD simulation for 75 ps to have a thermal equilibrium between the two regions. We set the MD simulation time step to 0.25 fs for all simulations. For every 6.25 fs time interval, we have recorded the configurations to analyze the transitions taking place between different bond topologies. The numbers attached to each arrow in Fig. 1 indicate the total number of registered transitions during the simulation in that direction between the complexes for a representative NC with a radius of $13.4 \AA$. The fact that there are almost balanced rates in opposite directions is an assurance of the attainment of the steady state in our simulation. Note that we do not observe any direct transition other than the paths indicated in Fig. 1. For instance, a direct transition of the complex $s s$ to $s m$ does not take place, but it is possible through an intermediate transition over the ssm, which is a $3 \mathrm{cO}$. We should also remark that the balanced transitions continue to take place after the steady state is attained, which indicates that the interface bond topology is dynamic, i.e., not frozen.

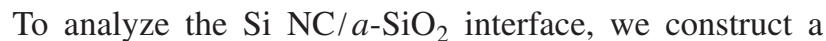
NC surface using the Delaunay triangulation scheme (Fig. 2 inset). ${ }^{15}$ In two dimensions, a Delaunay triangulation of a set of points corresponds to defining triangles such that no point in the set is inside the circumcircle of any triangle. In three dimensions, triangles extend to tetrahedra and circumcircles become circumspheres. Since our NCs are nearly spherical in shape, we triangulate projection points of surface $\mathrm{Si}$ atoms onto the unit sphere. Hence, we apply Delaunay triangulation over the two dimensional $\theta-\phi$ plane. We can then create surfaces using this triangulation. This surface enables us to calculate every atoms' distance to the NC surface. By this means, we plot various data such as charge, bond order, etc., with respect to distance to the surface to extract information 


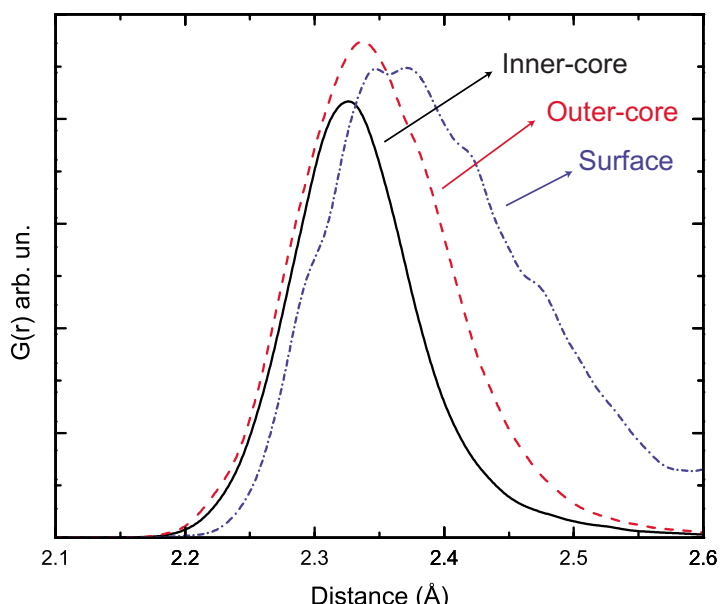

FIG. 3. (Color online) Bond distance probability distribution of $\mathrm{Si}$ atoms in NC. The solid line represents inner-core $\mathrm{Si}-\mathrm{Si}$ bonds, the dashed line outer-core $\mathrm{Si}-\mathrm{Si}$ bonds, and the dotted line surface $\mathrm{Si}-\mathrm{Si}$ bonds.

about the surface chemistry of Si NC embedded in amorphous matrix.

\section{RESULTS AND DISCUSSIONS}

For different NC radii, we observe similar trends in bond order distribution, average charges, etc.; therefore, we present only the figures of the system for a typical $\mathrm{NC}$ of radius $13.4 \AA$. Useful data to elucidate the structure of these systems are the radial distribution function (RDF). In Fig. 2, we present the RDF of $\mathrm{NC}$ atoms only, where the first broad peak centered at around $2.34 \AA$ with a $0.3 \AA$ full width at half maximum value (FWHM) represents the $\mathrm{Si}-\mathrm{Si}$ bond length distribution in NC (Fig. 2 inset). The maximum extent of the NC can also be read from same plot at about $27 \AA$, where the RDF goes to zero. Observation of a broad first peak in Fig. 2 demands further investigation of $\mathrm{Si}-\mathrm{Si} \mathrm{RDF}$ of NC atoms. For this purpose, in Fig. 3 we present bond length probability distributions (akin to RDF) for three categories of Si atoms: inner-core (with no bonds to surface atoms), outer-core (bonded to the surface), and surface NC atoms. We observe from Fig. 3 that $\mathrm{Si}-\mathrm{Si}$ bond lengths in the inner core are centered around the equilibrium value and have a narrow width due mainly to thermal vibrations, whereas the bond length distributions of outer-core and surface atoms have increasing shifts for the most probable bond length values and broader widths. These shifts, and particularly, the increase in distribution widths cannot be attributed to thermal broadening. Taken together, these two observations is a clear indication of increasing strain as a function of distance from the center of the NC. To further investigate this deviation of $\mathrm{Si}-\mathrm{Si}$ bonds from crystalline $\mathrm{Si}$, in Fig. 4 we present bond length distribution with respect to the distance to the NC surface averaged over 2 ps of simulation time after the steady state is reached. This figure illustrates the gradual development of radial strain from the center to the NC surface. These observations show clearly that oxidation at the

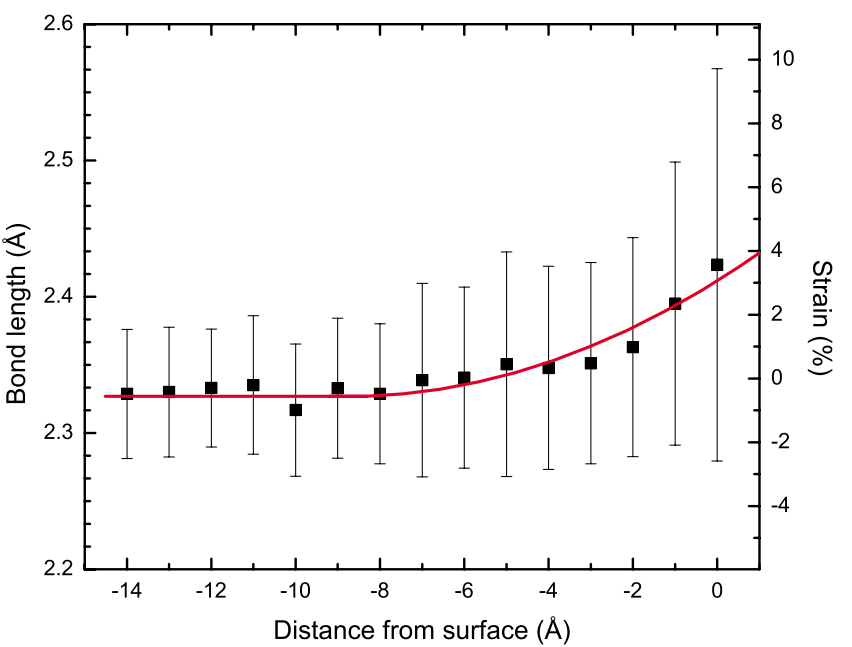

FIG. 4. (Color online) Variation of $\mathrm{Si}-\mathrm{Si}$ bond length averages (calculated over $1 \AA$ wide bins) as a function of distance from the NC surface, which is defined by Delaunay tesselation. The solid line is a fit to the data to guide the eye.

surface of $\mathrm{NC}$ results in a tensile strain at $\mathrm{Si}-\mathrm{Si}$ bonds, which becomes significant only around the interface, while keeping the inner core almost unstrained. This tensile strain in the NC agrees with previous measurements of Hofmeister et al. ${ }^{16}$

Another consequence of this tensile strain is that the total bond orders of core-NC atoms are somewhat smaller than those of oxide $\mathrm{Si}$, as seen in Fig. 5. In the same figure, we also show the calculated net charges using the electron equilibration method. ${ }^{17}$ Nearly zero net charges of the core-Si atoms reflect the covalent type of bonding well within the NC. The bonding becomes increasingly ionic away from the $\mathrm{NC}$ core, as observed by the charges of $\mathrm{Si}$

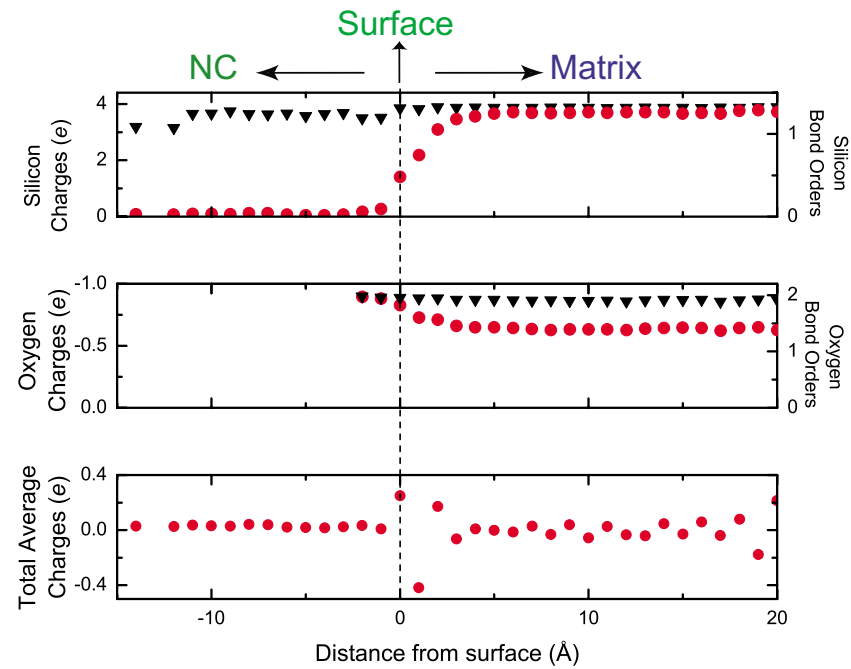

FIG. 5. (Color online) Top: Silicon bond orders (triangles) and charges (circles) as functions of distance from the surface of NC. Middle: Oxygen bond orders and charges as functions of distance from the surface of NC. Bottom: Total average charge as a function of distance from the surface of NC. The averaging bin width is $1 \AA$. 
TABLE I. Statistical results of atom charges and numbers $N$ for all NC diameters $D_{\mathrm{NC}}$ considered. The abbreviations for atom types are explained in Fig. 1. Charges are in the units of electronic charge and the angles $\theta$ are in degrees.

\begin{tabular}{|c|c|c|c|c|c|c|c|c|c|c|c|c|c|c|c|}
\hline \multirow{2}{*}{$\begin{array}{l}D_{\mathrm{NC}} \\
(\AA)\end{array}$} & \multirow[b]{2}{*}{$N_{c}$} & \multirow[b]{2}{*}{$N_{s}$} & \multirow[b]{2}{*}{$N_{O}$} & \multicolumn{3}{|c|}{ ss } & \multicolumn{2}{|c|}{$s m$} & \multicolumn{3}{|c|}{ ssm } & \multicolumn{2}{|c|}{ sss } & \multicolumn{2}{|c|}{$m m s$} \\
\hline & & & & $N_{s s}$ & Charge & $\theta_{s s}$ & $N_{s s}$ & Charge & $N_{s s m}$ & Charge & $\theta_{s s}$ & $N_{s s s}$ & Charge & $N_{m m s}$ & Charge \\
\hline 11.0 & 10 & 25 & 32 & 2 & -0.88 & 169.7 & 24 & -0.76 & 5 & -0.74 & 97.0 & 0 & & 1 & -0.81 \\
\hline 15.4 & 42 & 42 & 59 & 5 & -0.77 & 136.1 & 51 & -0.74 & 1 & -0.73 & 82.7 & 0 & & 2 & -0.77 \\
\hline 18.0 & 83 & 62 & 77 & 11 & -0.82 & 120.5 & 56 & -0.74 & 1 & -0.83 & 141.2 & 0 & & 9 & -0.79 \\
\hline 19.8 & 114 & 76 & 82 & 14 & -0.82 & 139.3 & 49 & -0.73 & 4 & -0.81 & 123.1 & 1 & -0.85 & 14 & -0.79 \\
\hline 26.8 & 353 & 143 & 170 & 20 & -0.81 & 118.0 & 123 & -0.74 & 11 & -0.79 & 120.9 & 0 & & 15 & -0.76 \\
\hline 30.8 & 558 & 203 & 243 & 35 & -0.83 & 123.9 & 159 & -0.74 & 10 & -0.80 & 115.9 & 2 & -0.80 & 34 & -0.79 \\
\hline 33.4 & 718 & 238 & 268 & 44 & -0.83 & 123.0 & 179 & -0.75 & 18 & -0.80 & 125.7 & 4 & -0.84 & 23 & -0.78 \\
\hline
\end{tabular}

atoms, which reach the value of $1.3 e$ at the oxide region (Fig. 5). As a result, the positive charges of surface-Si atoms form a shell at the surface of NC. This observation is similar to those obtained with DFT calculations. ${ }^{18,19}$ On the other hand, negative charges of oxygen atoms bonded to the surface form another shell that encloses NC, and, finally, total average charges approach to zero within the oxide region. In Fig. 5, we also observe that the magnitude of the average charges of oxygen atoms that are bonded to the surface is greater than that in the matrix. However, the bond orders are nearly the same. This is due to the existence of $3 \mathrm{cO}$ atoms bonded to the surface. Note that the average bond order of oxygen atoms that are bonded to the surface is about 2 (see Fig. 5). Thus, those oxygen atoms form three partial bonds, two strong bonds and one weak bond. Finally, we would like to note that unlike many others, ${ }^{2,3,6}$ we do not observe any double bonds.

The occurrence of $3 \mathrm{cO}$ has been noted by a number of groups. Pasquarello showed that the bistable $E_{1}^{\prime}$ defect of an $\alpha$-quartz structure may lead to $3 \mathrm{cO}$ as a metastable state as well as a $\mathrm{Si}-\mathrm{Si}$ dimer bond and calculated the energy of the former to be higher than the latter. Pasquarello proposed that $3 \mathrm{cO}$ acts as an intermediate metastable state during structural relaxations at the interface. ${ }^{20}$ Similarly, Boero et al. observed $3 \mathrm{cO}$ atoms in their $a b$ initio calculations ${ }^{21}$ and reported this feature as a metastable state. In Table I, we present the collected statistical data at the end of the simulation for $75 \mathrm{ps}$. For all oxygen complexes, the number of bridges, average charges of bridge oxygens, and average bridge angles for $s-\mathrm{O}-s$ are tabulated. We observe in Table I that the number of sss complexes is very small due to the narrow bond angle requirement of this configuration. For the remaining $3 \mathrm{cO}$ complexes, ssm and $\mathrm{mms}$, their percentages are seen to increase with curvature. This can explain the fact that other studies ${ }^{20,21}$ that have identified the $3 \mathrm{cO}$ complexes as metastable were based on the planar $\mathrm{Si} / \mathrm{SiO}_{2}$ interfaces. So, this is an indication of the importance of curvature in the stability of $3 \mathrm{cO}$ complexes. Hence, as one would expect, there is a linear relation between the total number of bridges with surface area, as indicated in Fig. 6. This finding is supported by Kroll and Schulte, who reported 3 and 33 such bridges for Si NC with radii 4.30 and $7.0 \AA$, respectively. ${ }^{18}$

\section{CONCLUSIONS}

In conclusion, the realistic chemical environment provided by the reactive force field model enables us to understand the bond topology of the $\mathrm{Si} \mathrm{NC} / a-\mathrm{SiO}_{2}$ interface and its internal dynamics. Particularly, it reveals that there are different types of oxygen complexes at the Si NC surface, some of which contain $3 \mathrm{cO}$ complexes whereas there are no double bonds. The curvature has a positive effect on the occurence of $3 \mathrm{cO}$. The relative abundance of different complexes and their charge and geometrical characteristics are extracted. The inner core is observed to be almost unstrained, while the outer core and the interface region of the $\mathrm{NC}$ are under increasing strain up to about a few percent. In general, our work clearly shows that the $\mathrm{Si} \mathrm{NC-oxide} \mathrm{interface} \mathrm{is}$ more complicated than the previously proposed schemes, which were based on solely simple bridge and double bonds. The provided information here paves the way to constructing realistic Monte Carlo moves for the simulation of large-scale silicon nanostructures embedded in an oxide matrix.

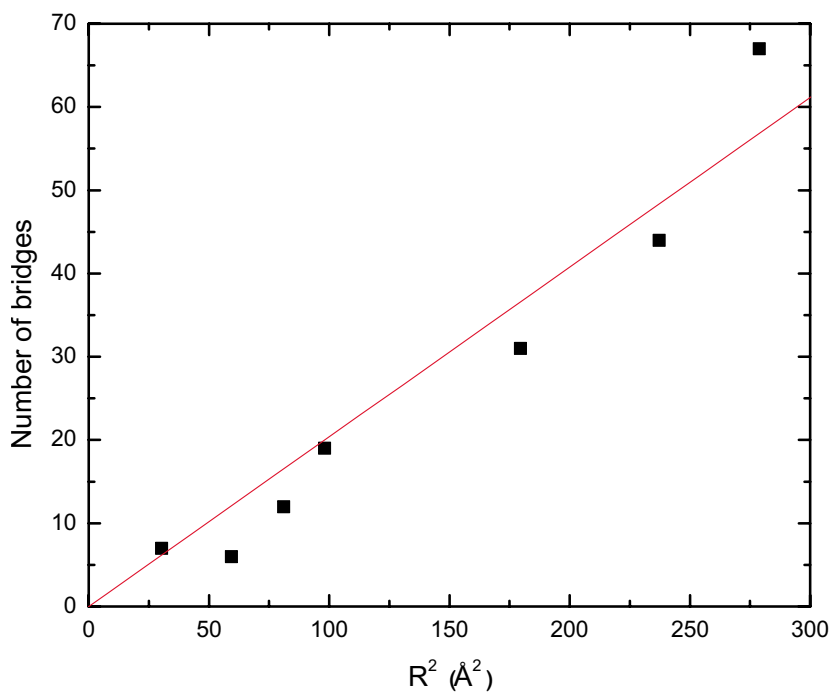

FIG. 6. (Color online) The number of bridges at the Si NC surface vs radius squared. The line is a linear fit to the data. 


\section{ACKNOWLEDGMENTS}

This work has been supported by the European FP6 Project SEMINANO with Contract No. NMP4 CT2004 505285 and by the Turkish Scientific and Technical Council
TÜBİTAK with Project No. 106T048. The visit of Tahir Çağın to Bilkent University was facilitated by the TÜBİTAK BIDEB-2221 program. The computational resources are supplied in part by TÜBİTAK through the TR-Grid e-Infrastructure Project. *dundar@fen.bilkent.edu.tr

†bulutay@fen.bilkent.edu.tr

*cagin@chemail.tamu.edu

${ }^{1}$ T. Canham, Appl. Phys. Lett. 57, 1046 (1990).

${ }^{2}$ M. V. Wolkin, J. Jorne, P. M. Fauchet, G. Allan, and C. Delerue, Phys. Rev. Lett. 82, 197 (1999).

${ }^{3}$ A. Puzder, A. J. Williamson, J. C. Grossman, and G. Galli, Phys. Rev. Lett. 88, 097401 (2002).

${ }^{4}$ E. Luppi, F. Iori, R. Magri, O. Pulci, S. Ossicini, E. Degoli, and V. Olevano, Phys. Rev. B 75, 033303 (2007).

${ }^{5}$ M. Gatti and G. Onida, Phys. Rev. B 72, 045442 (2005).

${ }^{6}$ I. Vasiliev, J. R. Chelikowsky, and R. M. Martin, Phys. Rev. B 65, 121302(R) (2002).

${ }^{7}$ Y. Tu and J. Tersoff, Phys. Rev. Lett. 89, 086102 (2002).

${ }^{8}$ G. Hadjisavvas and P. C. Kelires, Phys. Rev. Lett. 93, 226104 (2004).

${ }^{9}$ D. E. Yılmaz, C. Bulutay, and T. Çağın, J. Nanosci. Nanotechnol. 8, 635 (2008).

${ }^{10}$ A. Pasquarello, M. S. Hybertsen, and R. Car, Nature (London) 395, 58 (1998).

${ }^{11}$ D. W. Brenner, Phys. Rev. B 42, 9458 (1990); D. W. Brenner, O.
A. Shenderova, J. A. Harrison, S. J. Stuart, B. Ni, and S. B. Sinnot, J. Phys.: Condens. Matter 14, 783 (2002).

${ }^{12}$ A. C. T. van Duin, A. Strachan, S. Stewman, Q. Zhang, X. Xu, and W. A. Goddard III, J. Phys. Chem. A 107, 3803 (2003).

${ }^{13}$ E. Demiralp, T. Çağın, and W. A. Goddard, Phys. Rev. Lett. 82, 1708 (1999).

${ }^{14}$ N. T. Huff, E. Demiralp, T. Çağın, and W. A. Goddard, J. NonCryst. Solids 253, 133 (1999).

${ }^{15}$ R. J. Renka, ACM Trans. Math. Softw. 22, 1 (1996).

${ }^{16}$ H. Hofmeister, F. Huisken, and B. Kohn, Eur. Phys. J. D 9, 137 (1999).

${ }^{17}$ A. K. Rappe and W. A. Goddard, J. Phys. Chem. 95, 3358 (1991).

${ }^{18}$ P. Kroll and H. J. Schulte, Phys. Status Solidi B 243, R47 (2006).

${ }^{19}$ P. Kroll and H. J. Schulte, Mater. Res. Soc. Symp. Proc. 958, 0958L07 (2007).

${ }^{20}$ A. Pasquarello, Appl. Surf. Sci. 166, 451 (2000).

${ }^{21}$ M. Boero, A. Oshiyama, P. L. Silvestrelli, and K. Murakami, Appl. Phys. Lett. 86, 201910 (2005). 